\title{
The anesthetic challenges of a diagnostic biopsy of a large posterior mediastinal mass causing severe obstruction at the carinal level- Case report
}

\author{
Christopher W. Liu*, Eileen Y Sim and Shah Shital Kumar Sharad \\ Department of Anesthesiology, Singapore General Hospital, Outram Road, Singapore
}

\begin{abstract}
There is abundant literature about the anesthetic management of anterior mediastinal masses. There is also a perception that posterior mediastinal masses pose less significant problems compared to anterior mediastinal masses. In our case report, we describe the successful anesthetic management for a patient with symptomatic airway obstruction from a posterior mediastinal mass that required mediastinoscopy. Our report demonstrates that posterior mediastinal masses can be as difficult to manage as anterior mediastinal masses. It also highlights the importance of multidisciplinary cooperation, adequate preoperative planning and anticipation of problems during anesthesia.
\end{abstract}

\section{Introduction}

Mediastinal masses may give rise to substantial anesthetic problems due to their proximity to the large vessels, the heart and the tracheobronchial tree. The most feared perioperative complication is cardiorespiratory collapse which can occur at any point during the surgery such as induction of anesthesia with the use of sedatives, hypnotics, neuromuscular relaxants, positive pressure ventilation, positioning and handling or manipulation of the mediastinal mass. There have also been reports of cardiovascular collapse during emergence as a result of tracheomalacia secondary to prolonged compression by the mediastinal mass [1-8].

In this case report, we discuss the anesthetic considerations and management of a young patient with a large posterior mediastinal mass compressing on the tracheobronchial tree resulting in significant respiratory embarrassment at rest. We also conducted a literature review on anesthesia for posterior mediastinal mass and present our learning points.

\section{Case report}

The following case report received approval from the Singhealth Institutional Review Board. An informed consent was obtained for use of anonymous description of the case report.

A 34-year gentleman was listed for mediastinoscopy and biopsy of large posterior mediastinal mass as a semi-emergent case. He presented to hospital with progressively worsening dyspnea over the last 5 months accompanied by orthopnea, wheezing, sputum production, intermittent fever and right-sided chest pain. This is on the background of a diagnosis of "asthma" 2 years ago and in the last 5 months, he presented to the emergency department for a total of 7 times during which he was treated with bronchodilators, steroids, antihistamines and antibiotics. His other past medical history included hypertension, dyslipidemia and smoking.
On examination, he was sitting up and complained of shortness of breath. This was worsened with lying down. He had a pulse oxygen saturation of $91 \%$ on $4 \mathrm{~L} / \mathrm{min}$ of oxygen delivered via nasal prongs. On auscultation, he had widespread wheezing. He was obese (BMI $33.3 \mathrm{~kg} / \mathrm{m}^{2}$ ) but had a relatively normal looking upper airway.

His blood results (FBC, Renal panel, coagulation panel) and chest roentgenogram were grossly normal. However, his pulmonary function test revealed a severe airway obstruction with no reversibility. His forced expiratory volume (FEV1) was $11 \%$ and the FEV1/FVC ratio was $36 \%$. His arterial blood gas showed a $\mathrm{PaO}_{2} 77.2 \mathrm{mmHg}$ and a $\mathrm{SaO}_{2}$ of $96.4 \%$ on $2 \mathrm{~L} / \mathrm{min}$ of oxygen delivered via nasal prongs.

A computed tomography (CT) scan was performed in view of the obstructive pattern shown in his pulmonary function test. The CT scan showed large posterior mass extending from the thoracic inlet to the gastro-esophageal junction who was displacing the esophagus laterally, compressing the posterior tracheal membrane and was encasing the bronchus intermedius and azygos vein. The right lung was hyperinflated due to the ball-valve effect from the mass. There was a complete right main bronchus occlusion and at the carina, the trachea measured 5x7 mm (Figures 1 and 2).

In view of the high risk of a complete airway obstruction under anesthesia, we discussed with the surgeons, respiratory physicians and oncologists about the options of 1) Pre-treatment with chemotherapy/ steroids, 2) Obtaining biopsy under local anesthetic, 3) Performing a mediastinoscopy under general anesthesia, 4) Bronchoscopic techniques.

Correspondence to: Christopher W. Liu, Department of Anesthesiology, Singapore General Hospital, Outram Road, Singapore, Tel: (+65) 63214605; E-mail: christopher.liu@mohh.com.sg

Key words: thoracic anesthesia, central airway obstruction, mediastinoscopy

Received: April 02, 2015; Accepted: April 29, 2015; Published: May 01, 2015 


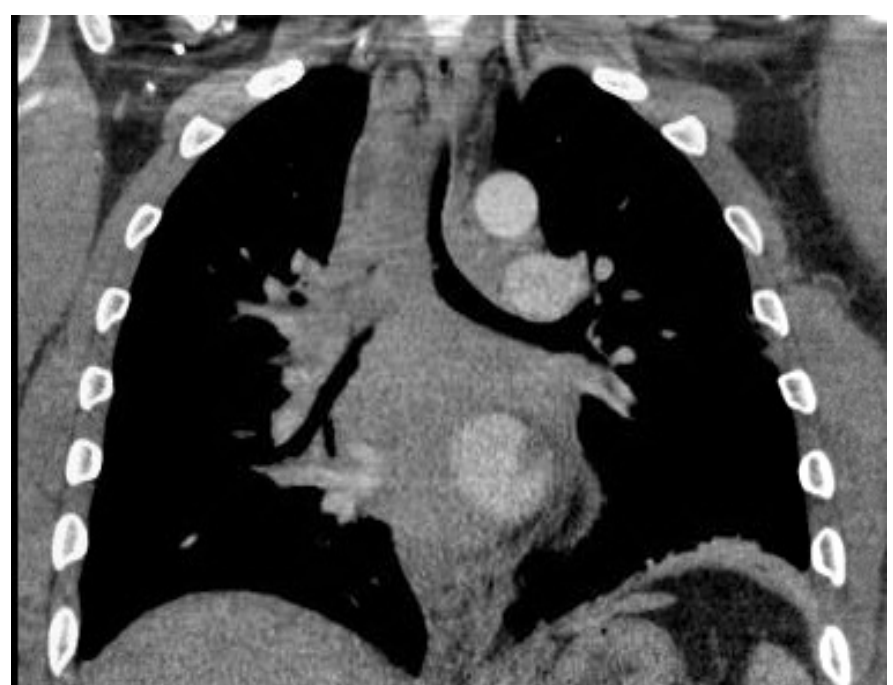

Figure 1. CT scan thorax PA view.

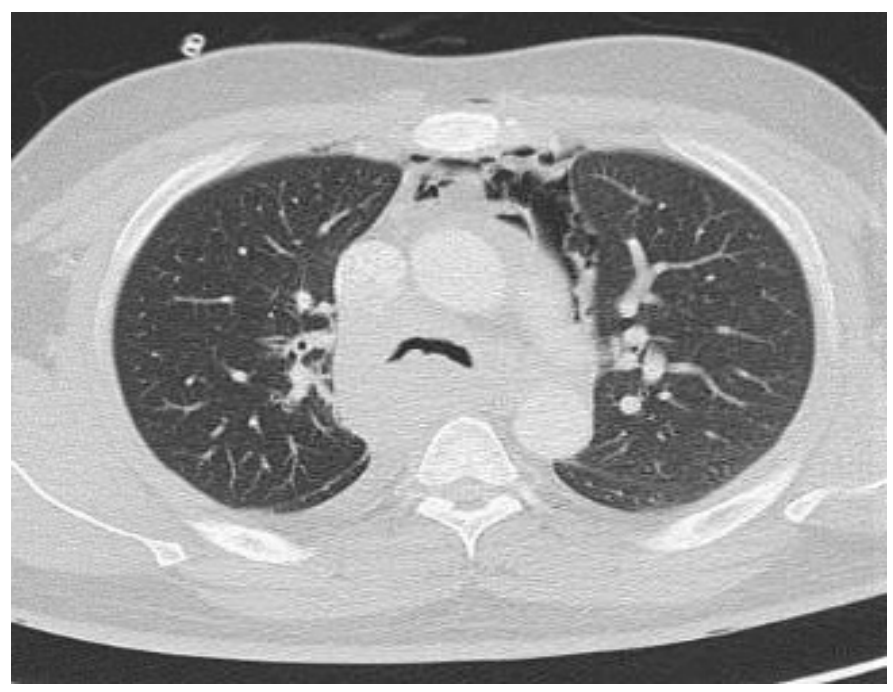

Figure 2. CT scan thorax: Transverse view.

After discussing the various options, the multidisciplinary team decided to obtain the biopsy by performing a mediastinoscopy. The oncologist was concerned that it may be difficult to obtain sufficient sample size for accurate histological diagnosis if pretreatment of chemotherapy or steroids given. There were no surgical sites available for obtaining biopsy under local anesthesia and we were worried that the patient was unlikely to tolerate a rigid bronchoscopy.

On close examination of the radiological investigations, we felt fairly confident of performing an endo bronchial intubation. Our plan was to perform an awake fiberoptic intubation of the left main bronchus and maintain spontaneous respiration throughout the surgical procedure. In the event of respiratory failure, we planned to initiate extra corporeal membrane oxygenation (ECMO). An intensive care bed was organized for post-operative phase.

\section{Anesthesia and intraoperative proceeding}

Standard monitoring (ECG, pulse oximetry, gas analyzers) and invasive arterial pressure monitoring were established on arrival of the patient to the operating room. The perfusionist team was in the operating room and the ECMO cart was primed and all equipment and trolley were kept ready. Groins were painted, draped and prepared for cannulation if required.

In sitting position, mouth and oral cavity was topically anaesthetized using $2 \%$ lignocaine nebulization and $10 \%$ lignocaine spray. The patient was given IV Midazolam $1 \mathrm{mg}$ and Fentanyl $25 \mathrm{mcg}$ for anxiolysis. In the sitting position, afiberoptic bronchoscope was advanced. At the carina, a large extra-tracheal mass was observed causing obstruction with patient breathing (Figure 3). We had selected a size-7 reinforced endotracheal tube (ETT) with a length of $29 \mathrm{~cm}$. However, even after inserting the full length of the ETT, the tip of the ETT was located $1 \mathrm{~cm}$ proximal to the carinal bifurcation. We decided to leave the ETT in that position as it appeared to be stenting the airway open.

Sevoflurane was used for induction and maintenance of anesthesia in order to preserve spontaneous respiration with PEEP of upto $5 \mathrm{~cm}$ water with view that PEEP might maintain airway patency against mass obstruction. The patient was then positioned supine with a shoulder. The surgeon requested for short duration of muscle paralysis so as to aid for maneuvering of medistinoscope. We commenced a remifentanil at an infusion rate of $0.1 \mathrm{mcg} / \mathrm{kg} / \mathrm{m}$ into provide acceptable surgical conditions. After the mediastinoscope was inserted, the tidal volume became shallow. We reduced the remifentanil infusion rate from 0.1 to $0.05 \mathrm{mcg} / \mathrm{kg} / \mathrm{min}$. We ruled out ETT blockage by suctioning the endotracheal tube and initiated hand ventilation and noted that the peak airway pressures rose to $50-60 \mathrm{mmHg}$ with only partially effective ventilation. However, we were able to maintain a $\mathrm{SpO} 2$ of more than $95 \%$. We suspected that this was due to a ball valve like effect secondary to the loss of negative intra thoracic pressure and a change in patient position.

The surgeons visualized a large tumor in the $4 \mathrm{R}$ region which they described a "fleshy" with a necrotic core. The frozen section showed that the tumor was lymphoid in origin. On completion of the surgery, anesthetic agents were discontinued and patient was returned to a sitting position. There was a period when the $\mathrm{SpO}_{2}$ fell to $85-90 \%$ and $\mathrm{EtCO}_{2}$ peaked to $70 \mathrm{~mm} \mathrm{Hg}$. This improved over next 10-15 minutes with the application of assisted positive pressure ventilation with $\mathrm{SpO}_{2}$ rising to more than $92 \%$ and $\mathrm{EtCO}_{2}$ to $55 \mathrm{~mm} \mathrm{Hg}$. After a further $10-15$ minutes, patient became more awake and appeared to have a cough

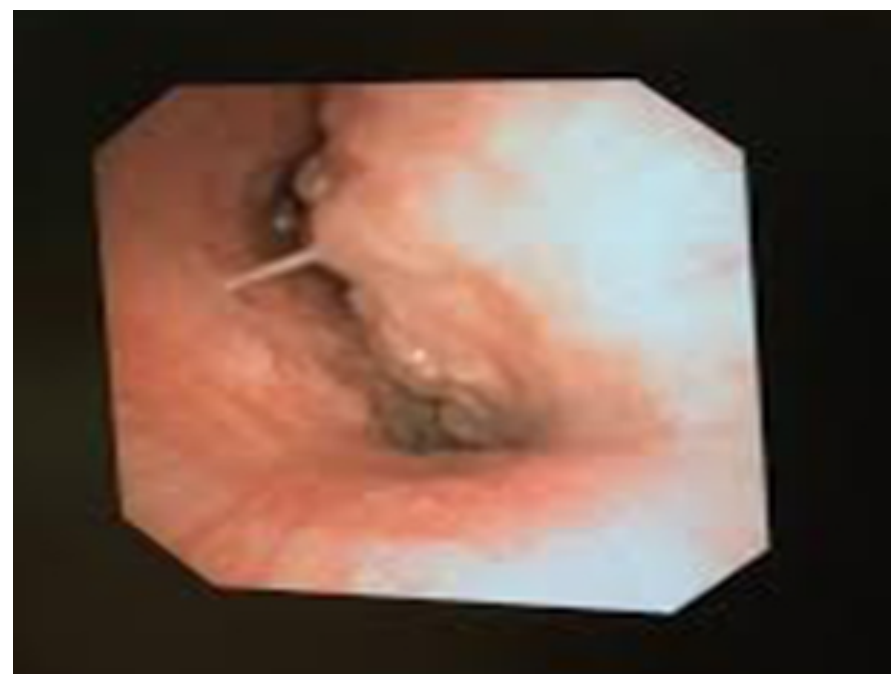

Figure 3. Fiberoptic bronchoscopy revealed a large extra-tracheal mass causing severe tracheal narrowing. 
reflex. We extubated at this point when $\mathrm{SpO}_{2}$ was more than $95 \%$ and $\mathrm{EtCO}_{2}$ below $55 \mathrm{~mm} \mathrm{Hg}$.

\section{Post-operative recovery}

Postoperatively, arterial blood gas on $50 \% \mathrm{FiO}_{2}$ in ICU showed hypoxia of $\mathrm{PaO}_{2}$ of $71 \mathrm{~mm} \mathrm{Hg}$. Chest roentgenogram performed postoperatively showed that the patient had developed a left pneumothorax and a chest tube was inserted. The patient needed noninvasive ventilation (NIV) overnight but was weaned off by the next morning. The patient was sent to the high dependency the next day and responded well to chemotherapy.

\section{Discussion}

The mediastinum is divided into a superior and inferior division. The inferior mediastinum can be further divided into the anterior, middle and posterior divisions. The posterior mediastinum is superiorly bounded by a plane between the sternal angle and T4. It is bounded by the diaphragm inferiorly, the pericardium anteriorly and the spinal cord posteriorly. The differential diagnoses of a posterior mediastinal mass are listed in Table 1 [9].

Many anesthesiologists consider the provision of anesthesia for patients with anterior mediastinal masses riskier than those with posterior masses as the weight of the anterior mediastinal mass may compromise the patency of the tracheobronchial tree, pulmonary artery and the right heart. Our case report demonstrates that the posterior mediastinal mass can result into similar problems as of anterior mediastinal mass. In the rest of the article, we will discuss about the differences between anterior and posterior mediastinal masses, multidisciplinary team effort, airway management and the role of extracorporeal membrane oxygenator.

\section{Differences between the anterior and posterior mediastinal mass}

Complete airway obstruction may occur during the delivery of general anesthetic to a high risk patient. This is attributed to the effects of a decreased lung volume, bronchial muscle relaxation and paralysis of the diaphragm which result in the reduction of an already compromised tracheobronchial diameter, greater compressibility of the trachea and elimination of the transpleural pressure gradient that acts as a physiological stent of the airway. As formerly mentioned, the weight of the anterior mediastinal mass may result in a more significant compression of the trachea during general anesthesia compared to the posterior mediastinal mass. However, since the effect of gravity is not the only determinant of airway diameter, there always remains a substantial threat of complete airway occlusion in a patient with a posterior mediastinal mass.

The anterior mediastinal mass may lie above the superior vena cava, the pulmonary artery or the right heart. Therefore, any of these structures may be compressed during the induction of anesthesia or

Table 1. Differential diagnosis of posterior mediastinal mass [9].

Neurogenic tumours (Schwanomma, Neurofibroma)

Neuroblastictumours (Neuroblastoma, Ganglioma)

Non-neurogenic tumours (Paraganglioma)

Paraspinal abscess

Descending aortic aneurysm

Oesophageal neoplasm

Hiatal hernias

Lymph nodes the perioperative period. In contrast, the posterior mediastinal mass is situated adjacent to the left heart. In particular, the left atrium is particularly prone to compression because of its inferoposterior position and increased compressibility compared to the left ventricle [10].

\section{Multidisciplinary effort}

A multidisciplinary team approach is vital for the perioperative care of patients with mediastinal masses. The parties involved may include the anesthesiologist, thoracic surgeon, respiratory physician, perfusionist, interventional radiologist, intensivist, nursing staff and theater assistants. Preoperatively, multidisciplinary team discussion is useful to decide: 1) Risk-benefit ratio of anesthesia and obtaining tissue for histology, 2) Approach to obtaining tissue for histology, 3) If general anesthesia is necessary, plan for securing airway, 4) Rescue measures in event of cardiorespiratory collapse

In our patient, the oncologist was worried that blind treatment of the tumor would result in missing the chance of obtaining a tissue diagnosis that will impact prognostication as well as future treatment for a young patient. We also considered the options of - rigid bronchoscopy, endobronchial ultrasound-guided biopsy and mediastinoscopy. After much discussion, it was decided that mediastinoscopy may be the safest approach considering definitive tissue biopsy sampling, our individual comfort level and because it is likely to cause the least trauma to the airway. We also felt that ventilation was possible if an endobronchial intubation was successfully performed.

\section{Airway management}

An individualized plan should be formulated for each patient based on his/her clinical presentation and radiological results. In particular, the CT thorax provides invaluable information. It has been suggested that patients can be risk stratified into [11-14.]

Low risk - Asymptomatic or mildly symptomatic, without postural symptoms or radiographic evidence of significant compression of structures;

Intermediate risk: Mild to moderate postural symptoms, tracheal compression $<50 \%$;

High risk-Severe postural symptoms, stridor, cyanosis, tracheal compression $>50 \%$ or tracheal compression with associated bronchial compression, pericardial effusion or SVC syndrome.

It would be prudent to avoid general anaesthesia in the high risk patient, using a local anaesthetic or local sedation for procedures such as biopsies [15]. If this is not possible, we recommend discussing with the oncologist about preoperative treatment of the mediastinal mass with steroids. It must be borne in mind though that the response to steroids may be unpredictable. It may precipitate tumour lysis syndrome and may shrink the tumor so much that subsequent tissue biopsy may not be sufficient for making a histological diagnosis [16].

In our patient, a peripheral lesion that was amenable to biopsy was not available. Therefore, we agreed to provide general anaesthesia for mediastinoscopy and biopsy of the posterior mediastinal lesion.

As mentioned before, the induction of general anesthesia may result in a complete obstruction of the airway, precipitating a cardiorespiratory collapse. We therefore considered two options: 1) Initiate ECMO from the start, 2) Perform an Awake fiberoptic endobronchial intubation to bypass the lesion and then use an anesthetic technique that will maintain spontaneous respiration throughout the surgery. 
We decided to perform the latter procedure because ECMO is not without risks. Firstly, anticoagulation is required during ECMO use. Secondly, there are risks arising from femoral vessel cannulation and systemic inflammatory response. Thirdly, it may be difficult to it may be difficult to provide subsequent treatment (e.g. radiotherapy) if the patient cannot be weaned off from ECMO support.

One of the problems that we faced was the availability of a specially designed endobronchial tube. The ideal endobronchial tube should be non-compressible (able to stent the airway), have a large enough internal diameter (decrease airway resistance to facilitate spontaneous respiration) and should be long enough. Double lumen tube could be difficult for two reasons: a: as the trachea has been narrowed to $5 \mathrm{~mm}$ by $7 \mathrm{~mm}$ and b: awake fibreoptic intubation with double lumen tube is more difficult than conventional single lumen tubes. As we did not have a specialized endobronchial tube, we used a size 7 Portex armored tube that had a length of $29 \mathrm{~cm}$. Unfortunately, at the level of the carina, the marking at the teeth was already $29 \mathrm{~cm}$. As a result, we had to accept a suboptimal position of the tube and proceeded with the surgery with the tube "splinting" open the airway.

As we were uncertain that the tube was able to completely stent open the airway, we chose to maintain spontaneous respiration during the surgery. This would allow maintenance of a transpleural pressure that will "splint" open the airway. Therefore, during the surgery, complete airway obstruction did not occur. However, the patient was noted to be hypo ventilating and we had to support his spontaneous breathing efforts with assisted positive pressure ventilation. The possible causes of his hypoventilation are:

1. Partial airway obstruction exacerbated by supine positioning and mediastinoscope

2. In complete expiration due to "ball-valve" effect from the tumor causing partial compression at the opening of the endotracheal tube.

3. Effect of low dose reminfentanil

4. Effect of volatile anesthetics

5. Increased work of breathing from breathing through the small diameter of the endotracheal tube.

\section{Extubation}

Extubation of a patient with a mediastinal mass is fraught with difficulties because the tube that is used to keep the airway open is about to be removed. In addition, inflammation from airway trauma may result in a decreased airway diameter compared to preoperatively.

In our case, we had caused minimal to nil airway trauma during the insertion of the endotracheal tube. Despite that, we used dexamethasone to reduce any inflammation that may have occurred. During extubation, we noted that the patient's $\mathrm{EtCO}_{2}$ was high. After much deliberation, we decided to trial extubation because we felt that the resistance of the endotracheal tube might be a significant contributing factor to the patient's decreased spontaneous ventilation efforts. This theory proved correct and the patient's EtCO2 and $\mathrm{PaCO}_{2}$ decreased markedly after extubation.

\section{Role of ECMO}

In our patient, collapse of the left atrium looked unlikely from the CT scan. However, the possibility of complete airway occlusion during induction of anesthesia was a real possibility.
Therefore, we had an ECMO primed and perfusionist on stand-by throughout the surgery.

We had a low threshold for using the ECMO if we:

1. Were unable to perform an endobronchial intubation

2. Were unable to "partially stent" the airway open with the PortexArmored Tube

3. Significant hypotension attributable to left atrial compression

Fortunately, we were able to complete the surgery without requiring need for ECMO. However, it must be warned that there are risks of not canulating the femoral vessels prior to initiating anesthesia and good patient selection is required. The literature is replete with recommendations of having ECMO on "standby" as a rescue measure, with the perfusionist's on hand and the machine primed. It has also been suggested that if ECMO is thought to be required, the femoral vessels should be cannulated before induction of anesthesia because the 5-20 min typically required to initiate ECMO may result in significant mortality and morbidity in the event of cardiorespiratory arrest [15-18]. Therefore, if the femoral vessels are not cannulated, we recommend a very low threshold for initiating ECMO before complete cardiorespiratory collapse occurs-so that salvage may be possible without the risk of developing end-organ damage.

\section{Conclusion}

In conclusion, we present the challenging case of a patient with a large posterior mediastinal mass with significant carinal obstruction which we manage to partially stent open with the endotracheal tube. Our case report highlights the difficulty with performing an endobronchial intubation in an adult because the standard endotracheal tube is not designed for endobronchial intubation. It also highlights the importance of using techniques that maintain spontaneous ventilation if an endotracheal tube is only able to partially stent the airway open. We also alert the problems with spontaneous respiration via a narrow endotracheal tube. We emphasize the reason for successful management due to a cooperative patient, competent specialists involved in the care, effective communication by the multidisciplinary team, proper planning, availability of experienced theatre staff and vigilant monitoring throughout the perioperative period.

\section{References}

1. Bray RJ, Fernandes FJ (1982) Mediastinal tumour causing airway obstruction in anaesthetised children. Anaesthesia 37: 571-575. [Crossref]

2. Ferrari LR, Bedford RF (1990) General anesthesia prior to treatment of anterior mediastinal masses in pediatric cancer patients. Anesthesiology 72: 991-995. [Crossref]

3. Levin H, Bursztein S, Heifetz M (1985) Cardiac arrest in a child with an anterior mediastinal mass. Anesth Analg 64: 1129-1130. [Crossref]

4. Mackie AM, Watson CB (1984) Anaesthesia and mediastinal masses. A case report and review of the literature. Anaesthesia 39: 899-903. [Crossref]

5. Neuman GG, Weingarten AE, Abramowitz RM, Kushins LG, Abramson AL, et al (1984) The anesthetic management of the patient with an anterior mediastinal mass. Anesthesiology 60: 144-147. [Crossref]

6. Northrip DR, Bohman BK, Tsueda K (1986) Total airway occlusion and superior vena cava syndrome in a child with an anterior mediastinal tumor. Anesth Analg 65: 10791082. [Crossref]

7. Pullerits J, Holzman R (1989) Anaesthesia for patients with mediastinal masses. Can J Anaesth 36: 681-688. [Crossref]

8. Robie DK, Gursoy MH, Pokorny WJ (1994) Mediastinal tumors--airway obstruction and management. Semin Pediatr Surg 3: 259-266. [Crossref]

9. Strollo DC, Rosado-de-Christenson ML, Jett JR (1997) Primary mediastinal tumors: 
part II. Tumors of the middle and posterior mediastinum. Chest 112: 1344-1357. [Crossref]

10. D'Cruz IA, Feghali N, Gross CM (1994) Echocardiographic manifestations of mediastinal masses compressing or encroaching on the heart. Echocardiography 11: 523-533. [Crossref]

11. Blank RS, de Souza DG (2011) Anesthetic management of patients with an anterior mediastinal mass: continuing professional development. Canadian journal of anaesthesia 58: 853-859, 860-857. [Crossref]

12. Erdös G, Tzanova I (2009) Perioperative anaesthetic management of mediastinal mass in adults. Eur J Anaesthesiol 26: 627-632. [Crossref]

13. Hack HA, Wright NB, Wynn RF (2008) The anaesthetic management of children with anterior mediastinal masses. Anaesthesia 63: 837-846. [Crossref]
14. Hammer GB (2004) Anaesthetic management for the child with a mediastinal mass. Paediatr Anaesth 14: 95-97. [Crossref]

15. Castillo M, Slinger P (2013) Myths of anterior mediastinal masses. Southern African Journal of Anaesthesia and Analgesia. 19: 38-40.

16. Borenstein SH, Gerstle T, Malkin D, Thorner P, Filler RM (2000) The effects of prebiopsy corticosteroid treatment on the diagnosis of mediastinal lymphoma. $J$ Pediatr Surg 35: 973-976. [Crossref]

17. Anderson DM, Dimitrova GT, Awad H (2011) Patient with posterior mediastinal mass requiring urgent cardiopulmonary bypass. Anesthesiology 114: 1488-1493. [Crossref]

18. Narang S, Harte BH, Body SC (2001) Anesthesia for patients with a mediastinal mass Anesthesiol Clin North America 19: 559-579. [Crossref]

Copyright: $(02015 \mathrm{Liu}$ CW. This is an open-access article distributed under the terms of the Creative Commons Attribution License, which permits unrestricted use, distribution, and reproduction in any medium, provided the original author and source are credited. 